\section{A case of emphysematous cystitis diagnosed by exploratory laparotomy}

\author{
Keisuke Takano, Hidetada Fukushima, \\ Yasuyuki Kawai, Yasuyuki Urisono, \\ Michiaki Hata, Kenji Nishio, \\ Kazuo Okuchi
}

Department of Emergency and Critical Care Medicine, Nara Medical University Hospital, Nara, Japan

\section{Abstract}

Emphysematous cystitis is a rare form of infection. Well known symptoms are: dysuria, urinary frequency and lower abdominal pain. We experienced a case of emphysematous cystitis presented with atypical peritoneal sign and computed tomography findings of massive intra-peritoneal fluid collection and abnormal gas appearance in pelvic space. Due to its presentation as acute abdomen, patient underwent exploratory laparotomy and the diagnosis of emphysematous cystitis was established. As far as we know, our case is the first report of emphysematous cystitis with intra-peritoneal fluid collection.

\section{Case Report}

An 83 year old female, who had been hospitalized for years due to schizophrenia, complained of vomiting and diarrhea for 2 days. She had no history of diabetes mellitus, neurogenic bladder, or previous antimicrobiotic therapy, but had a urinary catheter in place for a considerable period since she was bedridden for many years. The diagnosis was acute enterocolitis and intravenous fluid was administered. After she developed a chill and lower abdominal pain with muscular defense, she was diagnosed as surgical acute abdomen and transferred to our emergency department.

On arrival, her body temperature was $38.2^{\circ} \mathrm{C}$, blood pressure was $150 / 70 \mathrm{mmHg}$ with a regular heart rhythm of 110 beats/min, and her respiratory rate was 22 breaths/min. Physical examination revealed tenderness over the lower abdomen with muscular defense, rebound tenderness, and loss of bowel sounds. Laboratory examination revealed a normal white blood cell count with elevated $\mathrm{C}$-reactive protein $(24.9 \mathrm{mg} / \mathrm{dL})$. Urinalysis was unremarkable. An X-ray of the abdomen showed gas collection in the pelvic cavity, and CT scans of the abdomen revealed intraperitoneal fluid collection and abnormal gas accumulation in the pelvic space. The distribution of this abnormal gas was between the abdominal wall and retroperitoneal cavity including the urinary bladder wall, and its association with the intraperitoneal cavity was unclear (Figure 1).

In accordance with the history, physical examinations, and radiological findings, the patient was diagnosed as acute peritonitis with possible perforation or penetration of the lower intestine, and exploratory laparotomy was performed. At laparotomy, severe inflammatory changes were observed in the peritoneum from just below the umbilicus to the lower abdomen (Figure 2). Ascites in the peritoneal cavity was slightly turbid, but without a stool smell and there was almost no intraperitoneal inflammation. Other abdominal structures were intact, however, the bladder was swollen. When the bladder wall was pushed from the peritoneal cavity side, an unusual crackling sensation was felt and purulent urine was excreted via the urethral catheter. Dull dissection of the retroperitoneal cavity toward the bladder wall showed markedly loose connective tissue around the bladder due to severe emphysematous change, and the boundary with the bladder wall was unclear. These findings indicated emphysematous cystitis. The patient was placed on $1.5 \mathrm{~g} /$ day of intravenous doripenem for broad antibiotic coverage. Afterwards, $K$. pneumoniae was isolated from cultures of peritoneal fluid, urine, and blood (Table 1). Antimicrobiotic therapy
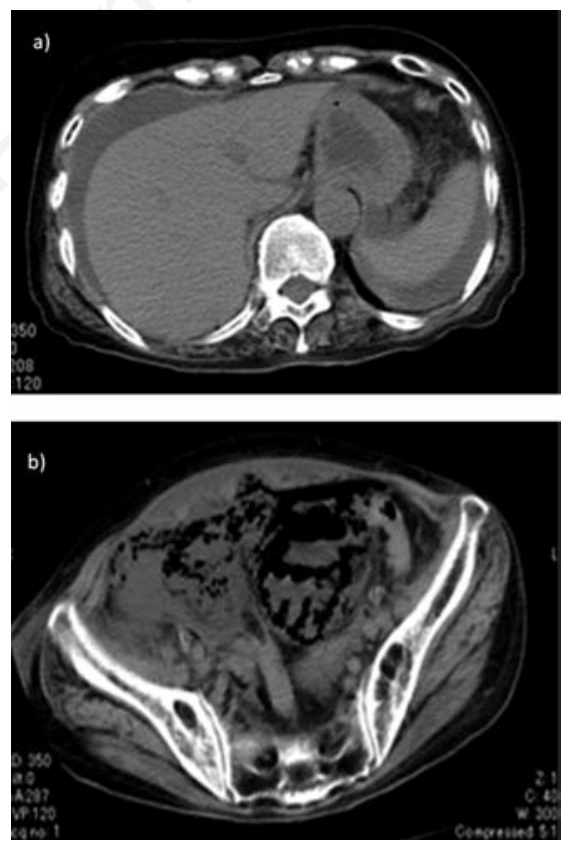

Figure 1. Abdominal computed tomography showing intra-peritoneal abnormal fluid collection (a), and gas accumulation in the pelvic space (b).
Correspondence: Hidetada Fukushima, Department of Emergency and Critical Care Medicine, Nara Medical University Hospital, 840 Shijo-cho, Kashihara, Nara 634-8522, Japan. Tel. +81.744.223.051 - Fax: +81.744.224.121.

E-mail: hidetakarina@gmail.com

Key words: emphysematous cystitis, exploratory laparotomy, acute abdomen.

Contributions: the authors contributed equally.

Conflict of interests: the authors declare no potential conflict of interests.

Received for publication: 26 December 2012. Revision received: 20 May 2013.

Accepted for publication: 24 May 2013.

This work is licensed under a Creative Commons Attribution NonCommercial 3.0 License (CC BYNC 3.0).

(C) Copyright K. Takano et al., 2013

Licensee PAGEPress, Italy

Infectious Disease Reports 2013; 5:e9

doi:10.4081/idr.2013.e

was changed to intravenous administration of ceftriaxone at $2.0 \mathrm{~g} /$ day for 2 weeks. The patient recovered uneventfully and follow-up CT imaging after 3 weeks demonstrated resolution of the bladder wall emphysema.
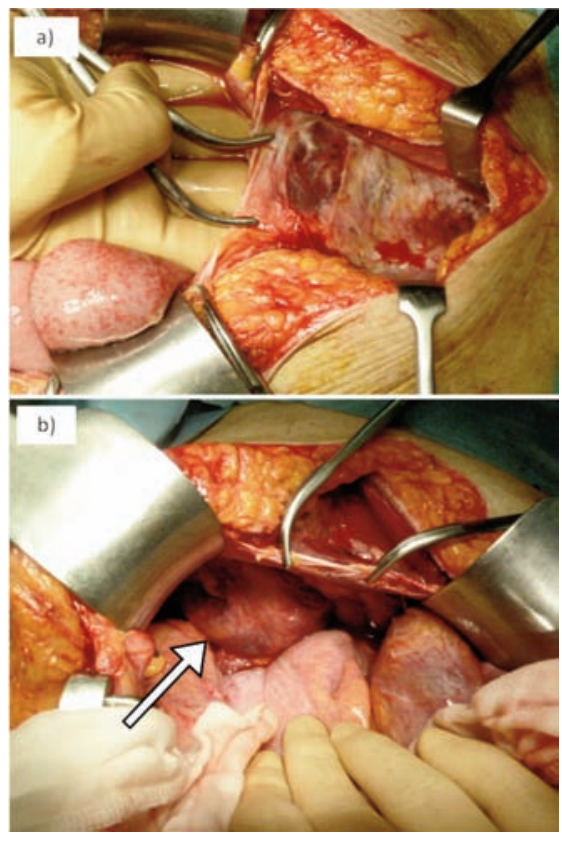

Figure 2. Exploratory laparotomy revealed inflamed peritoneum just below the umbilicus (a), and swollen bladder (b, arrow). 


\section{Discussion}

Emphysematous cystitis (EC) is a rare form of infection. There have been only 150 case reports so far. ${ }^{1}$ Several risk factors, such as diabetes mellitus, advanced age, neurogenic bladder, urethral catheter placement, vesicorectal fistula, end-stage renal disease, or outlet obstruction have been associated with development of this unusual form of infection. The most prevalent pathogens are $E$. coli and

Table 1. Antimicrobial susceptibility profile of isolated K. Pneumoniae.

\begin{tabular}{lc} 
Antimicrobial drug & MIC $(\mathrm{mg} / \mathrm{L})$ \\
Ampicillin & 16 \\
Amoxilin clavulanic acid & $\leq 2$ \\
\hline Cefazoline & $\leq 4$ \\
Cefotaxime & $\leq 1$ \\
\hline Cefepime & $\leq 1$ \\
Gentamicin & $\leq 1$ \\
\hline Levofloxacin & $\leq 0.12$ \\
Ciprofloxacin & $\leq 0.25$ \\
\hline Meropenem & $\leq 0.25$ \\
Sulfamethoxazole & $\leq 20$ \\
\hline
\end{tabular}

K. pneumoniae. ${ }^{1,2}$ The clinical presentation of this disease shows a wide spectrum, from asymptomatic to septic shock. Among the various symptoms, abdominal pain is relatively more common than classic symptoms of urinary tract infection (dysuria, urinary frequency, or urgency). However, frank peritoneal signs are less common. ${ }^{1,2}$

A typical radiographic finding of EC is abnormal gas accumulation within the bladder wall. It is reported that the generation of gas can result from the following: infection by anaerobes, the excretion of fermentation materials such as sugar, protein, and reduction in local blood flow. ${ }^{3}$ Although most cases can be diagnosed by X-ray or CT scan, it is rare that cases require laparotomy to establish the diagnosis. We have presented a case that underwent laparotomy because of acute abdominal pain, peritoneal signs, intra-peritoneum abnormal fluid collection, and gas accumulation in the pelvic cavity due to the possibility of perforation or penetration of the lower intestine. This laparotomy revealed a swollen bladder wall with emphysematous change and other abdominal structures intact. As far as we can determine, this case is the first report of EC with intra-peritoneal fluid collection.

Chong et al. also reported a case of EC that underwent exploratory laparotomy due to an abdominal finding of pneumoperitoneum. ${ }^{4}$
Pneumoperitoneum can develop as a result of air leakage into the peritoneal cavity thorough perforation of an inflamed bladder wall with emphysematous change.

Surgical procedures are rarely needed for the management of EC. Most cases can be managed by the administration of broad-spectrum antibiotics. ${ }^{1,2}$ However, due to its atypical presentation, this rare form of urinary tract infection may present as acute abdomen and require exploratory laparotomy to establish the diagnosis.

\section{References}

1. Thomas AA, Lane BR, Thomas AZ, et al. Emphysematous cystitis: a review of 135 cases. BJU Int 2007;100:17-20.

2. Grupper M, Kravtsov A, Potasman I. Emphysematous cystitis: illustrative case report and review of the literature. Medicine (Baltimore) 2007;86:47-53.

3. Huang JJ, Chen KW, Ruaan MK. Mixed acid fermentation of glucose as a mechanism of emphysematous urinary tract infection. J Urol 1991;146:148-51.

4. Chong SJ, Lim KB, Tan YM, et al. Atypical presentations of emphysematous cystitis. Surgeon 2005;3:109-12. 